\title{
PHOTOSPHERIC ABUNDANCES IN CENTRAL STARS OF PLANETARY NEBULAE, AND EVOLUTIONARY IMPLICATIONS
}

\author{
ROBERTO H. MENDEZ \\ Instituto de Astronomía y Física del Espacio \\ C.C. 67 , Suc. 28 \\ 1428 Buenos Aires, Argentina
}

\begin{abstract}
The available information about spectral characteristics and photospheric abundances of 115 CSPN is presented. About $33 \%$ of CSPN in the sample are H-deficient. Many (but not all) H-deficient CSPN show extremely high $\mathrm{C}$ abundances. There is also some evidence of a variety of $\mathrm{C}$ abundances among $\mathrm{H}$-rich CSPN. The available evidence suggests that most CSPN leaving the AGB with a H-rich photosphere will become DA white dwarfs. The "late He shell flash + fast wind" (born-again) mechanism may offer a satisfactory way of explaining some of the H-deficient CSPN. However, at least in some cases, the complete ejection of the H-rich stellar envelope, and exposure of He-burned matter at the remnant stellar surface, must happen already at the time of departure from the AGB.
\end{abstract}

\section{Introduction}

Central stars of planetary nebulae (CSPN) are hot pre-white dwarfs $\left(T_{\text {eff }}>20000 \mathrm{~K}\right)$ that have recently (less than 100000 years ago) ejected a substantial amount of mass (about half a solar mass, in order of magnitude) at rather low expansion velocities $(\leq 50 \mathrm{Km} / \mathrm{s})$.

By studying the photospheric abundances of CSPN, we can expect (a) to produce observational constraints on theoretical descriptions of the PN ejection process (which is extremely difficult to model), and (b) to check (or guide) predictions about what should happen to surface abundances as CSPN move along their evolutionary tracks and enter the white dwarf cooling tracks. In the end, we would like to be able to understand in full detail the variety of chemical abundances found among the Asymptotic Giant Branch (AGB) stars, planetary nebulae, CSPN and white dwarfs, and the relations between them, as functions of the nuclear burning, mixing and mass loss processes both on the AGB and after PN ejection, including gravitational settling in the more advanced stages. Of course, we are quite far from achieving this ambitious goal. The purpose of this review is to describe what is known about the photospheric abundances of CSPN, and what evolutionary constraints can be derived at the present time.

\section{First-order view of $P N$ ejection and post-AGB evolution}

After the work of Paczynski (1971) we have a widely accepted standard picture, which is very useful as a starting point for the discussion of pre-white dwarf stages in the evolution of intermediate-mass stars. A recent review is given by Schönberner (1989). Briefly, a star 
in a double-shell-burning phase climbs the AGB, losing mass at an increasingly higher rate, until the H-rich envelope mass is reduced below a certain limit. When this happens, the star (assumed to be burning $\mathrm{H}$ quiescently) leaves the AGB and moves at constant luminosity (given by the core mass - luminosity relation) towards higher surface temperatures. The mass lost in the latest AGB stages is ionized, becoming a PN, and later dissipates, while the star enters a white dwarf cooling track. When gravitational settling forces all heavier elements to sink, the star becomes a DA white dwarf.

This simple picture appears to be adequate, to first order, for the majority of CSPN and white dwarfs. However, a closer look uncovers several problems which have not yet been convincingly solved. The problem of post-AGB timescales (discrepancies with the kinematic nebular ages) is not directly related to the subject of this review; please refer to papers by Méndez et al. (1988a), Renzini (1989), Pottasch (1989) and McCarthy et al. (1990). Next, we have the problem posed by the existence of non-post-AGB CSPN, which requires the following introductory paragraph.

The positions of CSPN in the HR diagram are still a subject of some controversy, as a consequence of uncertainties in the classical determinations of stellar temperatures, distances and luminosities by indirect methods. However, according to their positions in the $\log \mathrm{g}-\log T_{\text {eff }}$ diagram, determined from direct spectroscopic studies, most CSPN are indeed post-AGB objects with typical white dwarf masses (Méndez et al. 1988a, Kudritzki and Méndez 1989). This conclusion is supported by careful studies of Magellanic Cloud PN (Barlow 1989). On the other hand, direct spectroscopic analyses have uncovered a few lowtemperature, high-gravity CSPN that cannot be post-AGB stars (Méndez et al. 1988b). The problem with these non-post-AGB CSPN (EGB 5, PHL 932) is the fact that they recently suffered substantial mass loss, which is not expected for such underluminous and (relatively) cool stars. Very probably, these non-post-AGB CSPN can only be understood as a consequence of a common-envelope phase in the evolution of close binary systems on the first red giant branch (Méndez et al. 1988b). Their photospheric abundances will be briefly discussed in Section 6.

Finally, we arrive to the problem that fills most pages of this review: many CSPN (and white dwarfs) are extremely $\mathrm{H}$-deficient. This is a problem because the simple picture described above requires departure from the AGB before the H-rich envelope is depleted by H-burning and mass loss. The suggested solution for this problem involves the removal of the H-rich envelope as a consequence of a late He-shell flash that sends the star back to the AGB (born-again phase) plus additional post-AGB mass loss: see e.g. Iben 1984, Renzini 1989 or Iben 1989 . I will come back to these ideas after describing the observational evidence about photospheric He abundance in CSPN.

\section{The basic framework: spectral classification of CSPN}

CSPN have always been difficult to study, for two main reasons: their faintness and the contamination of their spectra with nebular emissions. However, the "spectroscopic revolution" produced by the proliferation of large telescopes and the advent of modern spectrographs with high-quantum-efficiency detectors has made it possible, among many other things, to obtain much better spectrograms of many CSPN. In this section I have collected spectral information about 115 CSPN. In order to organize the presentation I have used a preliminary revised spectral classification scheme, already introduced in Méndez et al. (1986).

Of the 4 factors that influence the spectral characteristics of a CSPN $\left(T_{e f f}, \log g\right.$, mass 
loss rate and surface abundances), there is ever-increasing evidence (some of which will be mentioned in Section 6) that mass loss rate is mainly determined by a combination of $T_{\text {eff }}$ and gravity: the closer the star is to the Eddington limit (defined as a line in the $\log \mathrm{g}-\log T_{\text {eff }}$ diagram along which radiation pressure due to electron scattering is exactly balanced by surface gravity), the stronger is the stellar wind and mass loss. Therefore, in principle we can expect that a three-parameter classification scheme (surface abundance, wind strength, temperature) will provide a suitable framework. I have not given much emphasis to temperature-dependent criteria, because given a sufficiently good spectrum it is possible to obtain quantitative information about temperature, which clearly is preferable.

Most CSPN can be readily assigned to one or the other of two families: H-rich (stellar $\mathrm{H}$ features clearly seen in the spectrum) and $\mathrm{H}$-deficient (stellar $\mathrm{H}$ carefully searched but not detected). By "carefully" I mean sufficient spectral resolution, high signal-to-noise ratio, and efficient background suppression (in order to overcome the problem of superimposed nebular emission lines, the smallest entrance aperture, if possible similar to the stellar seeing disk, should be preferred).

I am currently working with the following definitions, adapted to the photographic and visual spectral regions:

\section{(a) H-rich CSPN:}

hgO(H): high-gravity stars, with very broad Balmer absorptions. These can be either non-post-AGB stars or stars positioned on white dwarf cooling tracks.

$\mathrm{O}(\mathrm{H})$ : stars showing spectra very similar to massive, young O-type stars, with He II 4686 in absorption. Spectral subtypes can be defined from the comparison between $\mathrm{He} I$ and He II absorptions, but excellent spectrograms are necessary for that purpose, because He I absorptions are easily masked by nebular emissions. It might be possible to refine the classification using $\mathrm{C}, \mathrm{N}, \mathrm{O}$ line ratios, but suitable criteria are not established yet.

Of $(H)$ : the stellar He II 4686 line is a narrow emission (FWHM $<4 \AA$; sometimes a P Cygni profile is seen). He II 4200, 4541 are in absorption, and are not blueshifted by more than 50 $\mathrm{Km} / \mathrm{s}$ relative to the nebular velocity. $H_{\gamma}$ is in absorption. For a more detailed discussion of this and the following class, see Méndez et al. 1990.

Of-WR(H): He II 4686 is a strong and broad emission, with FWHM $>4 \AA . H_{\gamma}$ shows an incipient or well developed P Cygni profile. He II 4541 is not in emission. When visible as an absorption, He II 4541 is substantially blueshifted, typically by about $100 \mathrm{Km} / \mathrm{s}$.

\section{(b) H-deficient CSPN:}

$\mathrm{O}(\mathrm{He}), \mathrm{O}(\mathrm{C})$ : stars showing predominantly an absorption line spectrum, dominated by the chemical element in parentheses.

Of(C), Of-WR(C): defined in a similar way as their H-rich counterparts, but of course using only the He II criteria. The spectrum is dominated by strong $\mathrm{C}$ emissions.

WC: defined in a similar way as the massive, Population I WC stars, with extensions to earlier and later subtypes. The criteria for subtype definitions are taken from Méndez and Niemela 1982 (WC2-WC6), van der Hucht et al. 1981 (WC7), Lundström and Stenholm 1984 (WC8-WC9), and Hu and Bibo 1990 (WC10-WC12). It is not clear if the latest subtypes (WC11-12) show photospheric Hydrogen or not; high-resolution spectrograms are not available, and it is difficult to decide if $\mathrm{H}$ emissions belong to the nebula or to the 
star. For the earlier subtypes, with broader stellar emissions, it is easy to check that there are no stellar $\mathrm{H}$ features, by comparing even- $n$ with odd- $n$ Pickering lines.

In Tables 1 and 2 , I have listed, respectively, $71 \mathrm{H}$-rich and $38 \mathrm{H}$-deficient CSPN. Table 3 gives 6 WC (or related) objects whose status as H-rich or H-deficient is not clear. At least $33 \%$ of the 115 objects are H-deficient.

TABLE 1 - H-RICH CSPN (71 OBJECTS)

\begin{tabular}{|c|c|c|c|c|c|c|c|c|}
\hline OBJECT & PK DESIGN & SP.TYPE & REF. & OBJECT & PK DESI & & SP.TYPE & REF. \\
\hline WDHS 1 & $\begin{array}{lll}197 & -6 & 1\end{array}$ & hgO(H) & 1,2 & NGC 6210 & $43+37$ & 1 & $O(H)$ & 21,22 \\
\hline HaWe 7 & $218-101$ & hgO (H) & 3,2 & NGC 6720 & $63+13$ & 1 & $O(\mathrm{H})$ & 23 \\
\hline HaWe10 & $211+181$ & hgO(H) & 3,2 & NGC 6804 & $45-4$ & 1 & $O(H)$ & 20 \\
\hline He $1-6$ & $224+11$ & hgO(H) & 4,2 & NGC 6853 & $60-3$ & 1 & $O(\mathrm{H})$ & 20 \\
\hline A 7 & $215-301$ & hgO (H) & 5,6 & NGC 7008 & $93+5$ & 2 & $O(H)$ & 24 \\
\hline A 31 & $219+311$ & hgO (H) & 7,6 & NGC 7094 & $66-28$ & 1 & $O(\mathrm{H})$ & 24 \\
\hline A 34 & $248+291$ & $\mathrm{hgO}(\mathrm{H})$ & 2 & H $2-1$ & $350+4$ & 1 & $O(H)$ & 10 \\
\hline A 39 & $47+42 \quad 1$ & hgO(H) & 7 & IC 2448 & 285-14 & 1 & $O(H)$ & 10 \\
\hline Lo 1 & 255-59 1 & hgO(H) & 8,6 & IC 3568 & $123+34$ & 1 & $03(\mathrm{H})$ & 25 \\
\hline EGB 6 & $221+461$ & hgO(H) & 9,2 & IC 4637 & $345+0$ & 1 & $O(\mathrm{H})$ & 10 \\
\hline NGC 7293 & $36-57 \quad 1$ & hgO (H) & 10 & He $2-138$ & $320-9$ & 1 & $O(H)$ & 10 \\
\hline IC 5148 & $2-521$ & hgO(H) & 6 & He 2-151 & $326-6$ & 1 & $O(H)$ & 10 \\
\hline EGB 5 & $212+231$ & hgo (H) & 10 & He 2-162 & $331-3$ & 1 & $\mathrm{O}(\mathrm{H})$ & 10 \\
\hline PHL 932 & $125-47 \quad 1$ & $\mathrm{hgO}(\mathrm{H})$ & 11 & He $2-182$ & $325-12$ & 1 & $O(H)$ & 10 \\
\hline A 15 & $233-161$ & $O(H)$ & 8,6 & K 2-15 & $263+0$ & 1 & $O(H)$ & 6 \\
\hline A 20 & $214+71$ & $O(H)$ & 6 & NGC 7009 & $37-34$ & 1 & $O(\mathrm{H})$ & 10 \\
\hline A 33 & $238+341$ & $\mathrm{O}(\mathrm{H})$ & 8 & \multicolumn{3}{|c|}{$C D-4113967$} & $O(H)$ & 26 \\
\hline A 36 & $318+411$ & $O(H)$ & 8,6 & \multicolumn{3}{|c|}{ IRAS $21282+5050$} & $O(H)$ & 27 \\
\hline A 41 & $9+101$ & $O(H)$ & 12 & IC 418 & 215-24 & 1 & $O f(H)$ & 10 \\
\hline A 43 & $36+171$ & $O(H)$ ? & 7 & IC 2149 & $166+10$ & 1 & of $(\mathrm{H})$ & 19,21 \\
\hline A 46 & $55+16 \quad 1$ & $O(H)$ & 7 & IC 4593 & $25+40$ & 1 & $05 f(H)$ & 25 \\
\hline A 51 & $17-101$ & $O(H)$ & 7 & NGC 2392 & $197+17$ & 1 & of $(H)$ & 10 \\
\hline Lo 8 & $310+241$ & $O(H)$ & 8,6 & NGC 5882 & $327+10$ & 1 & of $(\mathrm{H})$ & 10 \\
\hline Lo 13 & $345+151$ & $O(H)$ & 8 & NGC 6629 & $\begin{array}{ll}9 & -5\end{array}$ & 1 & $O f(H)$ & 10 \\
\hline LSE 125 & $335+121$ & $O(H)$ & 10 & NGC 6826 & $83+12$ & 1 & $03 f(H)$ & 25 \\
\hline LSS 1362 & $273+61$ & $O(H)$ & 13 & NGC 6891 & $54-12$ & 1 & of (H) & 10 \\
\hline LSS 2018 & $283+91$ & $O(H)$ & 14 & He 2-5 & 264-12 & 1 & of $(\mathrm{H})$ & 28 \\
\hline- & $242-371$ & $O(H)$ & 15,6 & He 2-108 & $316+8$ & 1 & of $(\mathrm{H})$ & 10 \\
\hline RWT 152 & & $O(H)$ & 16,17 & He 2-131 & $315-13$ & 1 & of $(H)$ & 10 \\
\hline Sa 4-1 & $75+351$ & $O(H)$ & 18 & M $1-26$ & $358-0$ & 2 & $O f(H)$ & 10 \\
\hline Sp 1 & $329+21$ & $O(H)$ & 10 & My Cn 18 & $307-4$ & 1 & of $(\mathrm{H})$ & 28 \\
\hline NGC 1360 & $220-531$ & $O(H)$ & 10 & Tc 1 & $\begin{array}{lll}345 & -8\end{array}$ & 1 & of $(H)$ & 10 \\
\hline NGC 1535 & $206-401$ & $O(H)$ & 10 & PB 8 & $292+4$ & 1 & of $-W R(H)$ & 10 \\
\hline NGC 3242 & $261+321$ & $O(H)$ & 10 & NGC 6543 & $96+29$ & 1 & Of $-W R(H)$ & 25 \\
\hline NGC 4361 & $294+431$ & $O(H)$ & 10 & NGC 6572 & $34+11$ & 1 & $0 f-W R(H)$ & 25 \\
\hline NGC 6058 & $64+48 \quad 1$ & $\mathrm{O}(\mathrm{H})$ & 20,19 & & & & & \\
\hline
\end{tabular}


REFERENCES IN TABLE 1:

1. Weinberger et al. 1983

2. Mendez, Ruiz, Herrero, Kunze (in prep.)

3. Hartl and Weinberger 1987

4. Weinberger 1977

5. Mendez et al. 1981

6. Mendez, Husfeld, Herrero, Kunze (in prep.)

7. Greenstein and Minkowski 1964

8. Mendez et al. 1985

9. Liebert et al. 1989

10. Mendez et al. 1988a

11. Mendez et al. 1988b

12. Green et al. 1984

13. Heber et al. 1988

14. Drilling 1985
15. Peña et al. 1990

16. Pritchet 1984

17. Chromey 1980

18. Sanduleak 1983

19. Herrero et al. 1990

20. Aller 1968

21. Heap 1977

22. McCarthy 1988

23. Kudritzki 1985 (priv.comm.)

24. Lutz 1977

25. Mendez et al. 1990

26. McCarthy et al. 1991

27. Cohen and Jones 1987

28. Mendez, unpublished

\section{Model atmosphere analyses of CSPN}

The determination of reliable photospheric abundances from the spectra of CSPN is complicated by three major difficulties: departures from local thermodynamic equilibrium (LTE), contamination of stellar features by nebular emissions, and (in some cases) the presence of strong stellar winds.

In the most favorable cases [spectral types $\mathrm{hgO}(\mathrm{H}), \mathrm{O}(\mathrm{H})$ and $\mathrm{Of}(\mathrm{H})$ ], nowadays it is possible to make a simultaneous determination of $T_{\text {eff }}, \log \mathrm{g}$ and surface He abundance, by fitting the observed stellar $\mathrm{H}$ and $\mathrm{He}$ absorption lines with theoretical line profiles obtained from plane-parallel, hydrostatic, metal-free ( $\mathrm{H}$ and $\mathrm{He}$ only) non-LTE model atmospheres in radiative equilibrium. The derived He abundances (which will be discussed in Section 6) have uncertainties of the order of $20 \%$ for solar values (Méndez et al. 1988a, 1988b).

A few preliminary analyses of H-deficient, absorption-line CSPN (K 1-27, NGC 246) have been made in a similar way: see Méndez et al. 1985, Husfeld 1987. Especially noteworthy is the very high $\mathrm{C}$ abundance (particle number fraction about 0.3 ) reported by Husfeld (1987) for the extremely H-deficient central star of NGC 246. More detailed analyses of $\mathrm{O}(\mathrm{He})$ and $O(C)$ CSPN are now possible after the work by Werner et al. (1990), who have studied four PG1159 stars using new non-LTE model atmospheres for mixtures of $\mathrm{He}, \mathrm{C}$ and $\mathrm{O}$. All these four very hot, high-gravity, H-deficient post-AGB stars, not surrounded by any $\mathrm{PN}$ (presumably because the $\mathrm{PN}$ have already dissipated), show very high $\mathrm{C}$ abundances (C/He about 0.5 by number, as in NGC 246) and also high $\mathrm{O}$ abundances (O/He about 0.13 by number). Another important recent paper is by Rauch et al. (1990), who have made a similar non-LTE study of the post-AGB star KS 292, which also lacks a PN and is interpreted as a He-burner showing in its photosphere a mixture of the original H-rich material with products of $\mathrm{H}$ - and He-burning. See also the review by Heber in this volume.

Hydrostatic, plane-parallel models are clearly useless for the analysis of Of-WR and WC CSPN. In such difficult cases, a more elaborate treatment of non-LTE radiation transfer for extended atmospheres with strong mass outflow is essential. Recent developments (Hillier 1989, Schmutz et al. 1989, Hamann et al. 1990, see also Gabler et al. 1989) are very encouraging. Hillier (1990) has suggested that in WC stars the absence of $\mathrm{H}$ lines requires $(\mathrm{H} / \mathrm{He})<0.1$ by number, and has reported (Hillier 1989) a $\mathrm{C} / \mathrm{He}$ number ratio of about 0.5 for early-type, Population I WC stars. It is reasonable to assume that WC CSPN have 
TABLE 2 - H-DEFICIENT CSPN (38 OBJECTS)

OBJECT PK DESIGN. SP.TYPE REF. OBJECT PK DESIGN. SP.TYPE REF.

\begin{tabular}{|c|c|c|c|c|c|c|c|c|c|}
\hline K $1-27$ & $286-29$ & 1 & $O(\mathrm{He})$ & 1 & NGC 6578 & 10 & -1 & WC early & 13 \\
\hline Lo $\operatorname{Tr} 4$ & $291+19$ & 1 & $O(\mathrm{He})$ & 2 & NGC 5189 & 307 & -3 & WC 2 & 15,16 \\
\hline K 1-16 & $94+27$ & 1 & $O(C)$ & 3 & NGC 2452 & 243 & -1 & HC 3 & 15,17 \\
\hline NGC 246 & $118-74$ & 1 & $O(C)$ & 4,5 & NGC 2867 & 278 & -5 & WC 3 & 15,16 \\
\hline Lo 3 & $258-15$ & 1 & $O(C)$ & 1 & NGC 6905 & 61 & -9 & HC 3 & 15,17 \\
\hline Lo 4 & $274+9$ & 1 & $O(C)$ & 1 & NGC 7026 & 89 & +0 & HC 3 & 15,17 \\
\hline vv 47 & $164+31$ & 1 & $O(C)$ & 6 & Sanduleak & 3 & & HC 3 & 16 \\
\hline IW 1 & $149-3$ & 1 & $O(C)$ & 7 & $\mathrm{He} 2-55$ & 286 & +2 & WC 3 & 18 \\
\hline Jn 1 & $104-29$ & 1 & $O(C)$ & 7 & NGC 5315 & 309 & -4 & WC 4 & 15,16 \\
\hline A 30 & $208+33$ & 1 & of $-W R(C) ?$ & 8 & NGC 6751 & 29 & -5 & HC 4 & 16 \\
\hline A 78 & $81-14$ & 1 & of $-W R(C) ?$ & 8 & IC 1747 & 130 & +1 & WC 4 & 15,17 \\
\hline $2-20$ & $0-1$ & 6 & WC & 9 & IRAS 1515 & $4-52$ & & HC 4 & 19 \\
\hline NGC 6567 & $11-0$ & 2 & WC & 14 & KPD $0721+$ & 0042 & & HC 4 & 20 \\
\hline M 3-15 & $6+4$ & 2 & WC & 14 & Cn $1-5$ & 2 & -9 & WC 4 & 9,18 \\
\hline A 58 & $37-5$ & 1 & WC early & 10 & IC 1297 & 358 & -21 & WC 4 & 18 \\
\hline M 3-30 & $17 \quad-4$ & 1 & WC early & 11 & NGC 40 & 120 & +9 & HC 8 & 17,21 \\
\hline IC 2003 & $161-14$ & 1 & WC early & 12 & $\mathrm{Pe} 1-7$ & 337 & +1 & HC 9 & 18,22 \\
\hline NGC 1501 & $144+6$ & 1 & WC early & 12 & $\mathrm{BD}+30 \quad 3639$ & 64 & +5 & WC 9 & 17,21 \\
\hline NGC 2371 & $189+19$ & 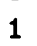 & WC early & 12 & He 2-99 & 309 & -4 & HC 9 & 23 \\
\hline
\end{tabular}

REFERENCES IN TABLE 2:

1. Mendez et al. 1985

2. Ref. 6 in Table 1

13. Kaler 1985

14. Aller and Keyes 1985

3. Grauer and Bond 1984

15. Mendez and Niemela 1982

16. Mendez et al. 1986

4. Heap 1975

17. Aller 1977

5. Kudritzki and Mendez 1989

18. Spectrum from Acker (priv. comm.)

6. Liebert et al. 1988

19. Manchado et al. 1989

7. Schönberner and Napiwotzki 1990

8. Cohen et al. 1977

20. Downes 1984

9. Webster 1975

21. Smith and Aller 1969

10. Seitter 1987

22. van der Hucht and Williams 1987

11. Kaler and Shaw 1984

23. Kaler et al. 1989

12. Heap 1982

\section{TABLE 3 - PROBABLY (BUT NOT NECESSARILY) H-DEFICIENT CSPN}

OBJECT

\section{SwSt 1}

He 2-113

M 4-18

CPD-56 8032

IRAS $17514-1555$

V $348 \mathrm{Sgr}$
PK DESIGN. SP.TYPE REFERENCES

$1-62$ Of-WR(C) Méndez, unpublished

$321+31$ WC 11 Webster, Glass 1974; Hu,Bibo 1990

$146+71$ WC 11 Goodrich,Dahari 1985; Hu,Bibo 1990

$332-91$ WC 11 Thackeray 1977; Hu,Bibo 1990

WC $11 \mathrm{Hu}$, Bibo 1990

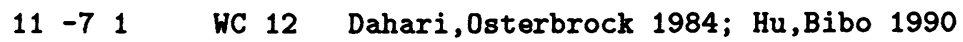


similar abundances, and it is extremely suggestive that the abundances of PG1159 stars are also similar (Werner et al. 1990).

\section{Heavier element abundances}

The already available high-resolution spectrograms of CSPN in the visual, photographic and UV (IUE) regions store a lot of information about heavier elements. Most of this information has not yet been adequately analyzed. A quantitative abundance analysis, requiring the solution of very complicated non-LTE line formation problems, is only now beginning to become possible for $\mathrm{C}, \mathrm{N}, \mathrm{O}, \mathrm{Mg}, \mathrm{Al}, \mathrm{Si}$. In the case of $\mathrm{Fe}$, nowadays it is possible only to report its presence or absence in the UV spectra of CSPN (Schönberner and Drilling 1985, Feibelman and Bruhweiler 1990).

The existence of a variety of $\mathrm{C}$ abundances among H-deficient CSPN is obvious [compare $\mathrm{O}(\mathrm{He})$ vs. $\mathrm{O}(\mathrm{C})$ central stars] even without accurate quantitative analyses. Although lack of space has forced me to decrease the number of figures to a minimum, I would like to report on some evidences implying a variety of $\mathrm{C}$ abundances also among the H-rich CSPN. The clearest case is provided by comparing the high-resolution CASPEC spectrograms of the central stars of NGC 1360 and NGC 4361. These two CSPN have almost the same $T_{\text {eff }}, \log \mathrm{g}$ and (normal) He abundance (Méndez et al. 1988a). The central star of NGC 4361 shows much stronger C IV absorptions $(4441,4646 \AA)$ and emissions $(4658,5801,5811$ $\AA)$. The simplest interpretation is that the photospheric $\mathrm{C}$ abundance in the central star of NGC 4361 is much higher. Coincidently, Torres-Peimbert et al. (1990) have presented an inhomogeneous model for NGC 4361, with an inner C-rich shell, that reproduces all the nebular $\mathrm{C}$ line intensities much better than homogeneous models.

There are other $\mathrm{H}$-rich CSPN showing rather strong C lines: e.g. IRAS 21282+5050 and IC 418. There is also one example of a C-deficient CSPN: the central star of NGC 2392 shows neither C III nor C IV features, together with very strong N III emissions and enhanced $\mathrm{He}$, suggesting the presence of $\mathrm{CNO}$-processed material at the photosphere.

\section{Evolutionary implications}

Figure 1 shows the $\log \mathrm{g}-\log T_{\text {eff }}$ diagram for 45 CSPN and 4 PG1159 stars. Figure 2 shows the photospheric $\mathrm{H}$ abundances (number fractions) as a function of $\log \mathrm{g}$. The data are taken from references $2,6,9,10,11,13,19,25$ and 26 in Table 1 , reference 1 in Table 2, Husfeld (1987) and Werner et al. (1990). Looking at the few H-deficient CSPN that can be plotted in Figure 1, it is important to note that they are, as expected, post-AGB stars. What about WC CSPN? A reasonable working hypothesis, according to the theory of radiatively driven winds, would be that they are all post-AGB stars, situated near the Eddington limit that corresponds to a H-deficient atmospheric composition. In practice, some doubts arise about the luminosities of WC CSPN, due to large uncertainties in the classical methods of PN distance determinations. My guess (extending conclusions obtained from the study of H-rich CSPN, see Méndez et al. 1988a, 1990) is that many "classical" distances are too small. Recent results by Masson (1989) bring support to this guess: his VLA expansion distances to BD+30 3639 (WC 9) and NGC 6572 [Of-WR(H)] are substantially larger than previously reported, and place these CSPN comfortably near the respective Eddington limit.

Warning: please do not read stellar masses for H-deficient CSPN in Figure 1, because they must be He-burners, which follow somewhat different evolutionary tracks (see Wood and Faulkner 1986, Koester and Schönberner 1986). 


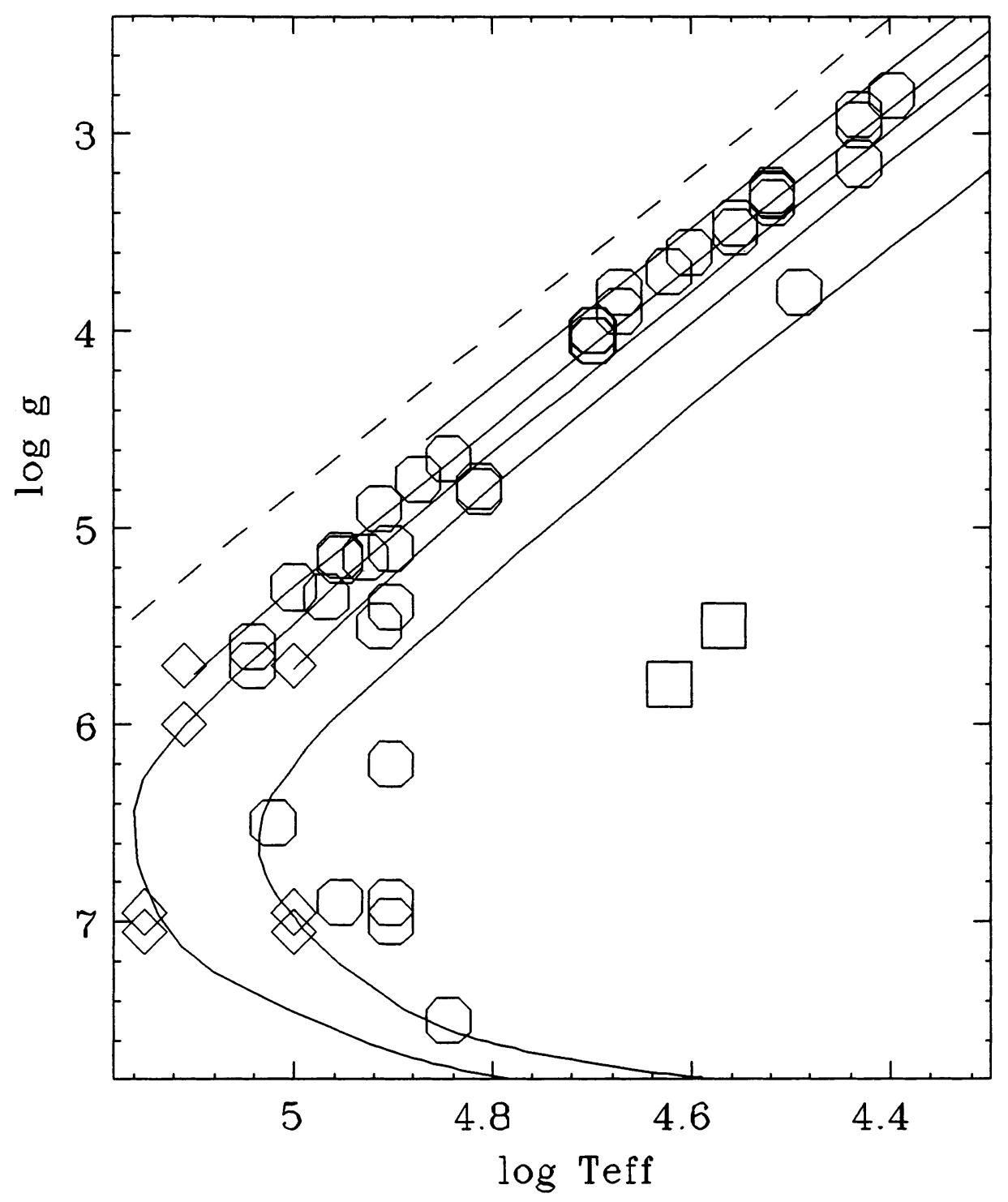

Figure 1. The $\log \mathrm{g}-\log T_{\text {eff }}$ diagram. The dashed line is the Eddington limit (defined in Section 3) for a "normal" photospheric He number fraction of 0.09 . The solid lines are post-AGB evolutionary tracks for H-burners of $0.76,0.644,0.598,0.565$ and $0.546 M_{\odot}$, taken from Wood and Faulkner (1986) and Schönberner (1989). I have forced a connection between two Schönberner tracks, for 0.598 and $0.546 M_{\odot}$, and the corresponding white dwarf cooling tracks computed by Koester and Schönberner (1986) for a $\mathrm{H}$ envelope mass of $0.0001 M_{\odot}$. The octagons are H-rich, post-AGB CSPN; the diamonds are H-deficient, post-AGB CSPN (the lower four are the PG1159 stars studied by Werner et al. 1990). The squares are two H-rich, non-post-AGB CSPN. 


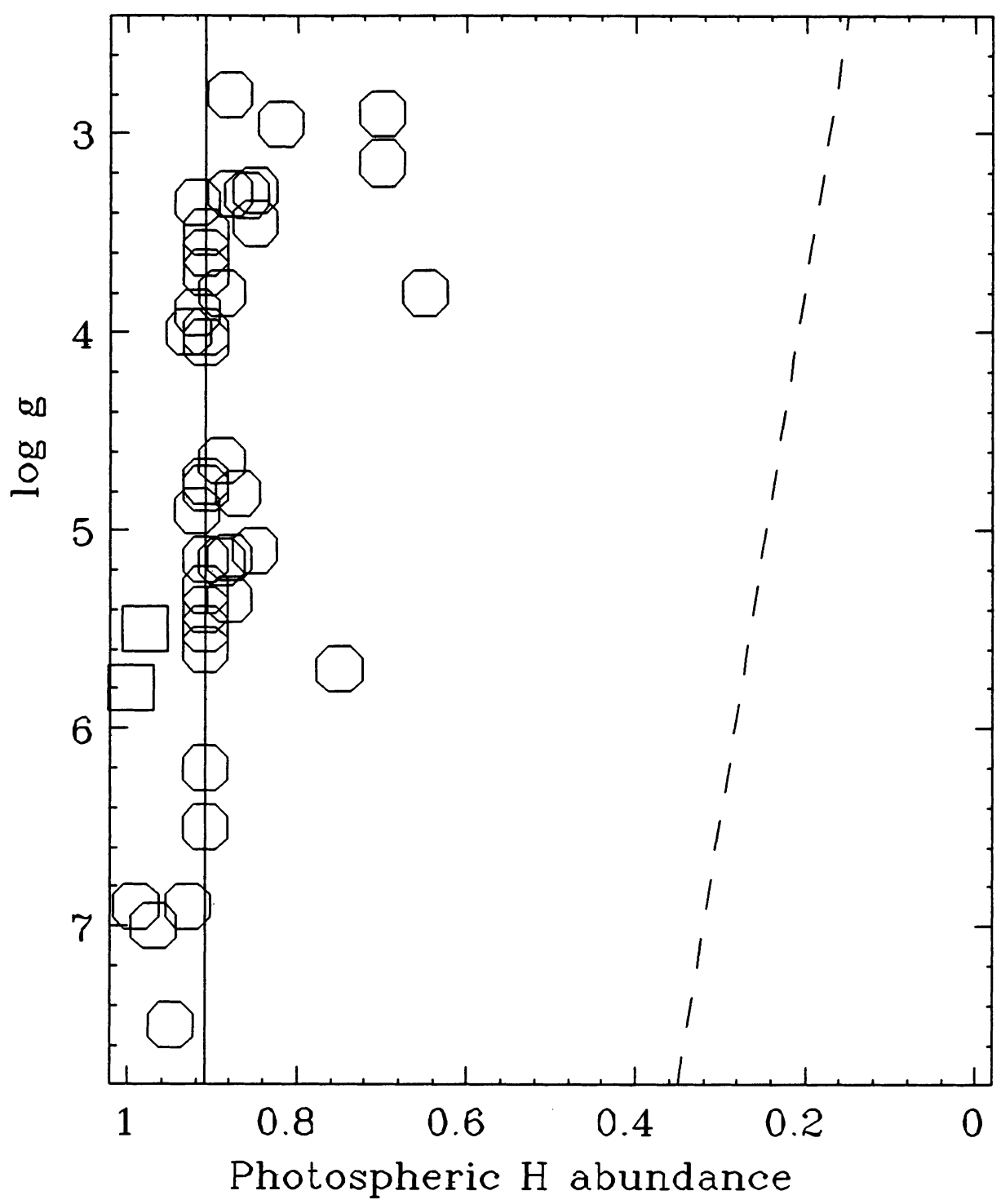

Figure 2. The photospheric $\mathrm{H}$ abundances (number fractions) of CSPN, plotted as a function of $\log \mathrm{g}$. The vertical solid line is the "normal" value, 0.91 . The inclined dashed line is a rough representation of the upper limit of the photospheric $\mathrm{H}$ abundance for $\mathrm{H}$-deficient CSPN. This upper limit is higher for higher temperatures and gravities. The symbols are the same as in Figure 1. Figure 2 shows clearly that there are very few CSPN with "intermediate" $\mathrm{H}$ abundances (less than 0.8 , more than 0.3 ). This conclusion would not change even if it were found that the Of-WR CSPN and the doubtful objects listed in Table 3 have such intermediate $\mathrm{H}$ abundances (which is probable but by no means certain). 
From the available information I would extract the following conclusions:

(a) CSPN with intermediate $\mathrm{H}$ abundances are infrequent. Besides, they are not more frequent at higher gravities. As far as I can estimate, the fraction of H-deficient CSPN is not a strong function of $T_{\text {eff }}$ or gravity, although cool H-deficient CSPN may be somewhat less frequent (this merits further investigation). In summary, for the moment we obtain rough agreement with the expectation that most CSPN leaving the AGB with a H-rich photosphere will become DA white dwarfs. This result is important in view of recent suggestions (e.g. Fontaine and Wesemael 1987) that the observed lack of very hot H-rich white dwarfs could mean that all CSPN become H-deficient along their post-AGB tracks. This idea is not supported by the available data. Indeed, notice in Figure 1 a few H-rich CSPN with normal He abundances and $T_{\text {eff }}>100000 \mathrm{~K}$. In addition, an apparent lack of very hot $\mathrm{H}$-rich white dwarfs may be produced, at least in part, by their faster evolution; H-burning CSPN suffer a fast luminosity drop, which allows them to arrive to the cooling tracks before the PN has had time to dissipate (He-burners evolve more slowly). In other words, many very hot, H-rich white dwarfs may be hidden within their PN.

(b) The two non-post-AGB CSPN show clear evidence of gravitational settling, as expected from their position in the $\log \mathrm{g}-\log T_{\text {eff }}$ diagram, far from the Eddington limit. Note that the much hotter H-rich post-AGB CSPN on the white dwarf cooling tracks show in some cases higher He abundances (below, but near, the solar value), implying perhaps that gravitational settling is not always overwhelming at temperatures above $70000 \mathrm{~K}$.

(c) The evolutionary connection between WC CSPN and PG1159 stars is becoming very convincing, in view of their similar $\mathrm{C}$ overabundances (but I still do not understand the lack of WC 5, 6 and 7 CSPN, already reported by Méndez and Niemela 1982 and Méndez et al. 1986). Concerning the origin of H-deficient CSPN, the "late He-shell flash + fast wind" (born-again) mechanism (Iben 1984, 1989) may offer in some cases a satisfactory explanation. However, in many other cases the loss of the remaining $\mathrm{H}$-rich material cannot be attributed to a fast wind acting along the He-burning post-AGB track; there is strong evidence requiring the complete ejection of the $\mathrm{H}$-rich envelope, and exposure of He-burned matter at the remnant surface, while the star is on the AGB. First of all, Iben (1984) has discussed a few cases (A 30, A 78) where ejection of H-deficient layers has happened at low expansion velocities, implying AGB precursors. Second, there are "cool" H-deficient CSPN like BD+30 3639, He 2-99 and NGC 40 (another example may be V348 Sgr, see Pollacco et al. 1990) already showing very high $\mathrm{C}$ abundances immediately after leaving the AGB. In connection with this, notice the lack of cool H-deficient CSPN showing predominantly absorption-line spectra; all cool $\mathrm{H}$-deficient CSPN are characterized by strong mass loss. This is not surprising, since AGB production of a $\mathrm{H}$-deficient CSPN can be expected to be more traumatic than AGB production of a H-rich one.

It is worthwhile to emphasize the existence of H-deficient CSPN surrounded by kinematically young nebulae: for example BD+30 3639, with a kinematic age of about 1100 years (Masson 1989), or the WC 4 central star of the small nebula NGC 5315. Iben (1984, 1989) explains that the best conditions for a quick exposure of $\mathrm{H}$-deficient layers are given when the late He shell flash happens on a white dwarf cooling track; in the case of BD+30 3639 , a rather massive post-AGB star of $0.8 M_{\odot}$ may be needed in order to arrive quickly enough to a sufficiently low luminosity before suffering the late He shell flash. However, this interpretation is not the only possible one; perhaps we should not yet rule out the possibility of producing a $\mathrm{H}$-deficient, $\mathrm{C}$-rich CSPN already at the time of first departure from the AGB, provided that the H-burning shell is not active when the PN ejection takes 
place.

One last comment: the variety of $\mathrm{C}$ abundances reported in Section 5 is an interesting problem for the future.

\section{References:}

Aller, L.H. 1968, in IAU Symp. 34 (Planetary Nebulae), p. 339.

Aller, L.H. 1977, R.A.S.C. Journal 71, 67.

Aller, L.H., Keyes, C.D. 1985, P.A.S.P. 97, 1142.

Barlow, M.J. 1989, in IAU Symp. 131 (Planetary Nebulae), p. 319.

Chromey, F.R. 1980, Astron. J. 85, 853.

Cohen, M., Hudson, H.S., O'Dell, S.L., Stein, W.A. 1977, M.N.R.A.S. 181, 233.

Cohen, M., Jones, B.F. 1987, Astrophys. J. 321, L151.

Dahari, O., Osterbrock, D.E. 1984, Astrophys. J. 277, 648.

Downes, R.A. 1984, P.A.S.P. 96, 807.

Drilling, J.S. 1985, Astrophys. J. 294, L107.

Feibelman, W.A., Bruhweiler, F.C. 1990, Astrophys. J. 357, 548.

Fontaine, G., Wesemael, F. 1987, in IAU Coll. 95 (The Second Conference on Faint Blue Stars), eds. A.G. Davis Philip et al., L. Davis Press, Schenectady, New York, p. 319.

Gabler, R., Gabler, A., Kudritzki, R.P., Puls, J., Pauldrach, A. 1989, Astron. Astrophys. 226, 162.

Goodrich, R.W., Dahari, O. 1985, Astrophys. J. 289, 342.

Grauer, A.D., Bond, H.E. 1984, Astrophys. J. 277, 211.

Green, R.F., Liebert, J., Wesemael, F. 1984, Astrophys. J. 280, 177.

Greenstein, J.L., Minkowski, R. 1964, Astrophys. J. 140, 1601.

Hamann, W.R., Wessolowski, U., Schwarz, E., Dünnebeil, G., Schmutz, W. 1990, in Properties of Hot Luminous Stars (Boulder-Munich Workshop), ed. C.D. Garmany, A.S.P. Conference Series 7, p. 259.

Hartl, H., Weinberger, R. 1987, Astron. Astrophys. Suppl. 69, 519.

Heap, S.R. 1975, Astrophys. J. 196, 195.

Heap, S.R. 1977, Astrophys. J. 215, 609.

Heap, S.R. 1982, in IAU Symp. 99 (Wolf-Rayet Stars), p. 423.

Heber, U., Werner, K., Drilling, J.S. 1988, Astron. Astrophys. 194, 223.

Herrero, A., Méndez, R.H., Manchado, A. 1990, in press.

Hillier, D.J. 1989, Astrophys. J. 347, 392.

Hillier, D.J. 1990, in Properties of Hot Luminous Stars (Boulder-Munich Workshop), ed.

C.D. Garmany, A.S.P. Conference Series 7, p. 340.

Hu, J.Y., Bibo, E.A. 1990, Astron. Astrophys. 234, 435.

van der Hucht, K.A., Conti, P.S., Lundström, I., Stenholm, B. 1981, Sp.Sci.Rev. 28, 227.

van der Hucht, K.A., Williams, P.M. 1987, The Observatory 107, 270.

Husfeld, D. 1987, in IAU Coll. 95 (The Second Conference on Faint Blue Stars), eds. A.G.

Davis Philip et al., L. Davis Press, Schenectady, New York, p. 237.

Iben, I. 1984, Astrophys. J. 277, 333.

Iben, I. 1989, in IAU Coll. 106 (Evolution of Peculiar Red Giant Stars), eds. H. R. Johnson and B. Zuckerman, Cambridge Univ. Press, p. 205.

Kaler, J.B. 1985, Astrophys. J. 290, 531.

Kaler, J.B., Shaw, R.A. 1984, Astrophys. J. 278, 195.

Kaler, J.B., Shaw, R.A., Feibelman, W.A., Lutz, J.H. 1989, Astrophys. J. Suppl. 70, 213. 
Koester, D., Schönberner, D. 1986, Astron. Astrophys. 154, 125.

Kudritzki, R.P., Méndez, R.H. 1989, in IAU Symp. 131 (Planetary Nebulae), p. 273.

Liebert, J., Fleming, T.A., Green, R.F., Grauer, A.D. 1988, P.A.S.P. 100, 187.

Liebert, J., Green, R., Bond, H.E., Holberg, J.B., Wesemael, F., Fleming, T.A., Kidder, K. 1989, Astrophys. J. 346, 251.

Lundström, I., Stenholm, B. 1984, Astron. Astrophys. Suppl. 56, 43.

Lutz, J.H. 1977, Astrophys. J. 211, 469.

Manchado, A., García Lario, P., Pottasch, S.R. 1989, Astron. Astrophys. 218, 267.

Masson, C.R. 1989, Astrophys. J. 346, 243.

McCarthy, J.K. 1988, Ph.D. Thesis, Caltech.

McCarthy, J.K., Mould, J.R., Méndez, R.H., Kudritzki, R.P., Husfeld, D., Herrero, A., Groth, H.G. 1990, Astrophys. J. 351, 230.

McCarthy,J.K.,Rich,R.M.,Becker,S.R.,Butler,K.,Husfeld,D., Groth,H.G. 1991,preprint.

Méndez, R.H., Groth, H.G., Husfeld, D., Kudritzki, R.P., Herrero, A. 1988b, Astron. Astrophys. 197, L25.

Méndez, R.H., Herrero, A., Manchado, A. 1990, Astron. Astrophys. 229, 152.

Méndez, R.H., Kudritzki, R.P., Gruschinske, J., Simon, K.P. 1981, Astron. Astrophys. 101, 323.

Méndez, R.H., Kudritzki, R.P., Herrero, A., Husfeld, D., Groth, H.G. 1988a, Astron. Astrophys. 190, 113.

Méndez, R.H., Kudritzki, R.P., Simon, K.P. 1985, Astron. Astrophys. 142, 289.

Méndez, R.H., Miguel, C.H., Heber, U., Kudritzki, R.P. 1986, in IAU Coll. 87 (H-deficient Stars and Related Objects), eds. K. Hunger et al., Reidel, Ap. Sp. Sc. Lib. 128, 323.

Méndez, R.H., Niemela, V.S. 1982, in IAU Symp. 99 (Wolf-Rayet Stars), p. 457.

Paczynski, B. 1971, Acta Astron. 21, 417.

Peña, M., Ruiz, M.T., Torres-Peimbert, S., Maza, J. 1990, preprint.

Pollacco, D.L., Tadhunter, C.N., Hill, P.W. 1990, M.N.R.A.S. 245, 204.

Pottasch, S.R. 1989, in IAU Symp. 131 (Planetary Nebulae), p. 481.

Pritchet, C. 1984, Astron. Astrophys. 139, 230.

Rauch,T.,Heber,U.,Hunger,K.,Werner,K.,Neckel,T. 1990, Astron.Astrophys., in press.

Renzini, A. 1989, in IAU Symp. 131 (Planetary Nebulae), p. 391.

Sanduleak, N. 1983, P.A.S.P. 95, 619.

Schmutz, W., Hamann, W.R., Wessolowski, U. 1989, Astron. Astrophys. 210, 236.

Schönberner, D. 1989, in IAU Symp. 131 (Planetary Nebulae), p. 463.

Schönberner, D., Drilling, J.S. 1985, Astrophys. J. 290, L49.

Schönberner, D., Napiwotzki, R. 1990, Astron. Astrophys. 231, L33.

Seitter, W.C. 1987, The ESO Messenger, No. 50, p. 14.

Smith, L.F., Aller, L.H. 1969, Astrophys. J. 157, 1245.

Thackeray, A.D. 1977, The Observatory 97, 165.

Torres-Peimbert, S., Peimbert, M., Peña, M. 1990, Astron. Astrophys. 233, 540.

Webster, B.L. 1975, M.N.R.A.S. 173, 437.

Webster, B.L., Glass, I.S. 1974, M.N.R.A.S. 166, 491.

Weinberger, R. 1977, Astron. Astrophys. Suppl. 30, 343.

Weinberger, R., Dengel, J., Hartl, H., Sabbadin, F. 1983, Astrophys. J. 265, 249.

Werner, K., Heber, U., Hunger, K. 1990, Astron. Astrophys., submitted.

Wood, P.R., Faulkner, D.J. 1986, Astrophys. J. 307, 659. 